CHRONIQUE DE L'ÉDITION SAVANTE

\title{
ÉDITIONS SAVANTES ET TECHNOLOGIE CONTEMPORAINE
}

L'édition d'œuvres complètes comme celles qui ont vu le jour dans les dernières décennies pose des problèmes spécifiques qu'il convient de traiter de préférence avant de se mettre à l'ouvrage. Comment, dans le contexte technologique de l'impression électronique, saisir les textes que l'on veut publier? Quel accompagnement leur donner pour que l'histoire de la philosophie armée trouve bénéfice dans l'édition savante? Ces réflexions concernent aussi bien les textes scientifiques que philosophiques, historiques que littéraires. Pour qui s'est trouvé responsable ou collaborateur d'entreprises aussi gigantesques que l'édition des $O C$ de Malebranche, de Spinoza, de Leibniz, de Cournot ou de Maine de Biran, une méthodologie finit par se dégager qui, face à l'invasion électronique et informatique, cherche à tirer le meilleur parti de cet "Informatikzeithalter $" 1$.

Notre connaissance de la culture des siècles précédents, qui aboutissait à la suprématie du livre, est en train d'effectuer un saut qualitatif. Ce saut qualitatif tient sa pertinence et son efficacité de l'introduction du quantitatif dans l'histoire de la philosophie et des sciences ${ }^{2}$. Cet effort quantitatif est parfaitement discernable sous deux aspects considérables. En ce qui concerne le XVII siècle :

- les éditions savantes de textes révèlent des écrits de plus en plus nombreux qui renouvellent notre connaissance du siècle ; les textes édités sont accompagnés et enrichis par un gros effort de publication scientifique des apparats critiques.

- la mise en orbite des applications de procédures informatiques fait pénétrer la quantité dans le maniement même de la documentation et de l'interprétation.

1. Cf. «Leibniz und Heidegger : Atomzeithalter oder Informatikzeithalter ? ", in Siudia leibnitiana, VIII-2, 1976, p. 241-256 ; Le Défi cybernétique : l'automate et la pensée, Paris, Gallimard, 1973 ( ( Les Essais », 179).

2. Voir la série des publications du Lessico Intelletuale Europeo ; "Peut-on introduire la quantité en histoire de la philosophie ? ", Actes du Colloque de Bruxelles sur Les Méthodes en histoire de la philosophie, 1974, publié in Philosophie et Méthode, Université libre de Bruxelles.

Revue de synthèse : IVe S. N 3, juillet-septembre 1986. 
Grâce à la convergence de ces procédés méthodiques, l'approche des textes philosophiques et scientifiques permet d'entrer dans l'époque d'une histoire de la philosophie armée, au sens où Claude Bernard entendait l'observation ou l'expérimentation armées.

Sur le premier point, je soulignerai d'abord l'extensivité prodigieuse du domaine inventorié.

D'une culture monoidéique centrée sur la notion de " siècle cartésien ", on est successivement passé à une culture multiidéique faisant appel au respect de la multiplicité des courants philosophiques et scientifiques du siècle. Il me semble que l'on aboutit aujourd'hui à une culture omniidéique, à organicité combinatoire, qui, à partir de l'observation armée ponctuelle d'un texte est capable de susciter la reconstitution d'une ambiance contrastée et conflictuelle globale.

C'est dire que le constat monoïdéique du « siècle de Descartes ", entraînant des divisions archétypales de pré-cartésien, avec Mersenne, Gassendi ou Galilée, de post-cartésien, où l'on a confondu pêle-mêle Malebranche, Spinoza et Leibniz, est maintenant entièrement suranné, même si des auteurs attardés s'y réfèrent encore.

La tendance multiidéique s'est développée sous la pression des années 50 avec la convergence des efforts génétiques et structuraux, conduisant des auteurs comme Gouhier, Garin, Gueroult ou Alquié à conférer au concept de " texte " une valeur intra-dimensionnelle qui a permis de récupérer la variété des textes dans le siècle et la diversité des doctrines ou des époques d'un auteur.

En passant de l'esprit de système à l'esprit $d u$ système, on régénérait l'attention nécessaire portée au principe d'individuation des doctrines : Leibniz n'était pas Descartes, Spinoza n'était pas Hobbes. Toute détermination devenant une négation, l'originalité des doctrines recevait sa pleine valeur singulière, de Mersenne à Newton, de Galilée à ses disciples, de Bacon à Arnauld, etc. Une culture multiidéique permettait d'expliquer les Méditations non seulement par les Réponses aux objections, mais aussi par les Objections mêmes. Quoiqu'une telle direction de recherche soit peu du goût de certains historiens du cartésianisme, les études sur les textes des scolastiques du XVII* siècle, sur Mersenne, sur Hobbes, sur Arnauld, sur Gassendi, sur les libertins, se sont largement développées dans toute l'Europe. Le propre des équipes du C.N.R.S. ou du C.N.R. a été de favoriser l'éclosion d'une nouveauté qui me conduit à proposer d'appliquer à ce gigantesque effort le terme de "renaissance " des études philosophiques et scientifiques au XVII siècle. Pour se rendre compte de cette multiplicité quantitative, de l'extension nouvelle des recherches en ce domaine, il n'est que de consulter le catalogue du Centro milanais ou du Lessico de Rome, ou les Recherches sur le XVII siècle de Paris.

Le concept même de " texte classique " s'en est trouvé renouvelé. Qu'est-ce qu'un " grand texte », que sont des " minores " ? La culture de base de la première moitié du XX' siècle s'est trouvée en ce domaine entièrement subvertie par ses propres progrès. Le renouvellement des conditions paléographiques, des conditions scientifiques d'édition, des conditions d'admission culturelle d'auteurs ou de textes ignorés ou répudiés, l'évolution des conditions idéologiques, ont permis cette extension du champ inventorié. 
Sur quoi s'appuie cette explosion quantitative des découvertes textuelles et de leur mise en culture ? Manifestement, tout commence par la recherche, la mise en valeur et la publication des textes. De partout nous viennent de nouvelles révélations grâce auxquelles le concept de « bon texte » est entièrement surpassé et laisse place au concept d'intégralité du discours textuel. Que l'on publie Malebranche ou le Theophrastus, que l'on s'intéresse à Bacon ou Fardella, qu'on en vienne à Galilée ou à Bayle, il faut d'abord constituer les niveaux du sol textuel que l'on interrogera. Le discours textuel entraîne la considération omnidimensionnelle des éléments rédactionnels du corpus considéré. La culture monoïdéique réduit le texte à la linéarité successive de son énonciation rhétoricologique. La culture multiidéique tient de plus en plus compte des conditions réelles de l'argumentation, sous l'effet de Perelman ${ }^{3}$, c'est-à-dire de l'impact sur le discours que peut avoir la considération du lecteur ou de l'auditeur ou du contradicteur. La culture omniidéique a pour objectif de faire sortir le texte des coordonnées linéaires ou spatio-temporelles pour le considérer dans l'épaisseur du discours, selon les strates de sa composition et selon les accidents de sa mise en forme. Ce qui condamne a priori comme nulle et non avenue pour le savoir toute édition anastatique de pure répétition éditoriale.

Quelles sont les conditions à remplir pour faire entrer le texte dans l'orbite omnidimensionnelle du discours ? Voici les quatre règles selon lesquelles organiser la tâche.

Règle I. Tous les manuscrits rapportés au corpus considéré doivent être compilés, leurs variantes recueillies et leurs stemma établis ; de même pour les imprimés. Cette règle de la globalité scripturaire entraîne qu'il n'y a rien d'indifférent dans la paléographie d'une œuvre et que son conditionnement matériel en doit être étudié scientifiquement ${ }^{4}$.

Un corollaire important de cette Règle I se prolonge aujourd'hui par la recherche sur les exemplaires singuliers des imprimés du XVII siècle, et a fortiori des siècles avoisinants, comme relevant non pas du texte constitué, mais du texte en composition. À la recherche classique des diverses éditions, il convient d'ajouter la discrimination des exemplaires d'une même édition, puisqu'il est avéré que l'auteur intervient sur les épreuves en cours de composition et que les tirages des imprimés s'effectuent sur des folios qui ont pu être remaniés. De plus la conservation de l'orthographe originale permet d'apprécier, en plus des phénomènes linguistiques, la situation culturelle de l'auteur ou de l'ouvrier.

La base scientifique de cette Règle I repose sur la saisie de l'ensemble des éléments rédactionnels qui confèrent au texte son épaisseur. Cette saisie s'appuie aujourd'hui sur d'importants procédés de laboratoire :

3. Cf. " Nouvelle rhétorique et informatique : le pouvoir dialectique ", dans le numéro en Hommage à Perelman, Rev. intern. de phil., 127-128, 1979, p. 129-144.

4. Nous en avions pris conscience sur les manuscrits de Leibniz en publiant notre premier ouvrage, Principes de la nature et de la grâce et Monadologie, d'après les manuscrits d'Hanovre, Paris, P.U.F., 1955. Cette édition cinético-cinématographique, qui en est à sa troisième édition, avait reçu les encouragements de P.-M. Schuhl. Nous avons appliqué cette règle à l'édition des OC de Malebranche et de Cournot, et il va de soi qu'elle reste la seule valable pour le continent des manuscrits leibniziens. 
- La science papier, provoque l'étude du matériau sur lequel les éléments du discours ont été enregistrés : étude des folios, des filigranes, des usines fabricatrices, des réseaux commerciaux, etc. Cette recherche est particulièrement féconde pour les vastes correspondances et pour leur datation, ou pour l'étude des fonds manuscrits importants et des brouillons (œuvre de Leibniz) ${ }^{5}$.

- La science graphique permet de préciser les moments de composition des manuscrits, les familles de recherches entreprises par l'auteur, etc.

- La science autographique par la considération des événements répertoriables sur les conditions de la composition du discours par l'analyse de contenu, les repérages bio-bibliographiques, l'établissement des datations, les garanties d'authenticité, les authentifications.

- La science autobiographique avec les confidences des auteurs sur les circonstances de la composition, les ouvrages consultés, les bibliothèques personnelles, les enseignements reçus, etc.

Règle II. L'apparat critique doit comporter intégralement toutes les leçons recueillies et les présenter de manière distincte et progressive, de façon à transformer la chronologie en diachronie. Deux façons de faire peuvent se présenter selon la nature du corpus assemblé.

- ou bien partir de la version originelle et présenter l'apparat avec les enrichissements successifs.

- ou bien conserver la version terminale et présenter l'apparat selon les diverses phases qui y aboutissent.

Quand les variantes d'une œuvre se présentent sur une longue période chronologique, il y a avantage à totaliser les versions en fonction du dernier état du texte et de faire de même pour tous les textes de façon à posséder les états terminaux simultanés du plus grand corpus possible : cette disposition devient très favorable pour l'étude de l'homogénéité des structures à un moment donné 6 . La différenciation génétique structurale en sera affinée et facilitée par la comparaison avec les états précédents, référables aux diverses " époques » de l'œuvre que cette analyse génético-structurale permet d'élaborer, cet établissement s'effectuant à partir de l'omnidimensionalité du discours et non pas en fonction de critères historiques ou herméneutiques externes.

Règle III. L'analyse de la multidimensionalité du discours ressortit à la statistique lexicographique et aux applications informatiques qui en sont faites pour faciliter et armer l'étude des textes. L'histoire armée des textes trouve aujourd'hui un adjuvant considérable par l'intervention

5. Nous nous y étions adonné en rassemblant et triant les pièces qui allaient conduire aux Mathematica, t. XVII-2, des OC de Malebranche; cf. l'emploi de ces méthodes classiques : " Le groupe malebranchiste introducteur du Calcul infinitésimal en France ", Revue d'histoire des sciences, XIII, 4, 1960, p. 287-308.

6. Nous nous sommes opposé à la façon dont Masson avait édité la Profession de foi du Vicaire savoyard, cf. " Lexicographie philosophique de NATURE dans la Profession ", Etudes sur le XVII' siècle, V, 1978, p. 23-42 ; " Lexicographie d'ORDRE DE LA NATURE dans la Profession ", Rev. intern. de phil., 124-125, 1978, p. 238-259 ; " Lexicographie et paléographie : à propos d'ORDRE dans la Profession ", Annali dell'Università di Napoli, Studi filosofici, I, 1978, p. 39-76. 
des moyens-calcul que permet directement l'enregistrement des textes sur bande.

Une première condition est remplie par la systématisation de la recherche documentaire, de la constitution des stemmas à celle des index de diverses typologies.

Une seconde condition est celle de l'enregistrement intégral du corpus considéré, codé avec ses déterminations diachroniques.

Une troisième condition consiste à relever les reliefs propres à l'enregistrement lexicographique, qui prépare la phase de distanciation sémantique. La poursuite de l'analyse sémiotique exige la suspension du sens. Cette déconstruction objectivante permet de tisser des rapports que l'on ne pourra passer sous silence dans l'approche sémantique.

Les index d'occurrences permettent de relever, dans n'importe quel corpus, les termes similaires et d'en conserver la référence au texte qui en permet la consultation. L'approche des concepts par la terminologie qui les connote est ainsi facilitée.

Les concordances qui suivent la série des occurrences donnent le contexte sur la dimension que l'on souhaite, du relevé linéaire au relevé du paragraphe. On glane ainsi tous les passages où se trouve inséré le terme cherché. Mais les concordances diacritiques sont plus performantes et recueillent les syntagmes plus ou moins lourds en fonction desquels fonctionne un terme considéré. On dévoile ainsi les structures lexicographiques d'assemblages qui font la redondance du discours et permettent d'étudier les variables de ces constellations lexicales.

Un certain nombre de procédures raffinées permettent de relever les dispositions des termes en pyramide avec à la pointe le terme pôle et vers la base les termes qui s'en trouvent de plus en moins rapprochés et plus ou moins fréquemment. Une autre sortie spectaculaire permet de repérer dans un corpus la " rafalité " des apparitions d'un pôle, la manière dont un terme se trouve plus ou moins dispersé dans une œuvre.

Ces reliefs lexicographiques mis en évidence, il appartient au commentateur d'en retenir le questionnement pour en exploiter les renseignements. Ainsi, l'approche sémiotique du texte apporte de premiers enseignements qu'il conviendra de ne pas perdre de vue dans le travail interprétatif 7 .

Règle IV. Selon les conditions techniques de l'édition électronique, il y a tout avantage à faire imprimer le texte sur les claviers à mémoire qui en conservent l'intégralité et qui permettent d'en tirer immédiatement et sans opération supplémentaire, les listes d'occurrences, les concordances et toute autre forme de classification répondant aux procédures automatisées. Une édition armée pourra être immédiatement pourvue des relevés lexicographiques diachroniques, qui font apparaître pour le commentateur les reliefs dont il faudra tenir compte. On

7. Nous avons consacré une cinquantaine d'articles à présenter ces procédures au fur et à mesure que nous les estimions rentables. D'un côté, nous avons fait paraître des textes classiques " armés » de ces résultats dans la collection « Philosophie et informatique ». De l'autre de gros volumes qui synthétisent ces résultats en attendant un équipement électronique plus maniable : l'Ethica de Spinoza, l'Index de Malebranche. 
obtiendra aussi immédiatement le relevé des noms propres, des noms d'auteurs, des noms de lieu, des références bibliographiques, etc.

C'est à nos centres de recherche de se procurer le matériel nécessaire, aujourd'hui banal, utilisable d'autre part pour la correction d'épreuves. Car l'ère de l'artisanat est entièrement dépassée pour la confection des éditions savantes : l'électronisation de l'impression et la puissance-calcul qui en permet l'exploitation changent radicalement les conditions de fabrication de l'édition savante et lui confèrent ses propres critères scientifiques dès le départ. Nous sommes entrés dans l'ère de l'au-delà du livre et le livre lui-même devient l'artefact des procédures informatiques aussi bien pour sa phase documentaire que pour sa phase heuristique. Nos centres de recherche doivent en donner l'exemple et les preuves, en développant ces méthodologies nouvelles.

On s'apercevra aussi que cette distantiation ne permet pas le retour à une simple herméneutique, un peu plus au fait des conditions concrètes de l'étude du discours. Le conditionnement sémiologique apporte son propre questionnement qu'il importe de poursuivre dans l'approche du sens. Il s'agit de conférer au sémiologique toute sa portée dans l'interprétation sémantique, de passer du non-sens au sens par la discrimination des structures inévitables du discours. $\grave{A}$ cette condition, on sortira de l'âge herméneutique.

Les fruits concernant la langue et le langage en sont considérables : spécificité des lexiques par matières, spécificité des vocabulaires d'auteurs ou des courants, néo-latinité et ses créations au XVIle siècle, étude comparative des langages naturels et des langues scientifiques ou philosophiques, etc. Et aussi, problème des traductions.

À ces conditions, l'organicité des œuvres retrouve sa solidité architectonique singulière. La quantité extensive et la quantité intensive confèrent à la méthodologie textuelle l'assurance que l'on peut mesurer en histoire de la philosophie ${ }^{8}$.

André RoBinet, Paris-Bruxelles.

8. Nous avons présenté et développé ces réflexions de part et d'autre du lac de Garde : en avril 1985 lors du colloque de Gargnano, "Le edizioni dei testi filosofici del ' 500 e del '600, Problemi di metodo et prospettive di ricerca ", organisé par le Centro di studi del pensiero filosofico de Milan ; en septembre 1985 lors du colloque de Trento, « Critical Editions and History of Mathematics ", organisé par le Centro internazionale per la ricerca matematica. 